\title{
Star Formation in Nearby Dwarf Galaxies*
}

\author{
S. S. Kaisin ${ }^{1 * *}$ and I. D. Karachentsev \\ ${ }^{1}$ Special Astrophysical Observatory, Russian Academy of Sciences, Nizhnii Arkhyz, 369167 Russia
}

(Received July 20, 2018; Revised December 12, 2018; Accepted December 12, 2018)

We report the measured $\mathrm{H} \alpha$ fluxes and images of 66 nearby objects observed with the 6-m telescope of the Special Astrophysical Observatory of the Russian Academy of Sciences. Three of these objects-IC 2233, UGC 4704, and NGC 3432-are late-type spiral galaxies, six objects are distant globular clusters of the M31 galaxy, and the remaining ones are dwarf galaxies. We used the measured $\mathrm{H} \alpha$ fluxes to estimate the integrated and specific starformation rates and analyzed some of the main features of star formation in dwarf galaxies and late-type spirals based on a sample of more than 500 Local-volume galaxies.

Keywords: galaxies, dwarf - galaxies, star formation

\section{INTRODUCTION}

Over the last decade observations of nearby galaxies aimed at determining their star-formation rate (SFR) from $\mathrm{H} \alpha$ emission flux have been carried out at the Special Astrophysical Observatory of the Russian Academy of Sciences. The $\mathrm{H} \alpha$ survey targets are Local-Volume (LV) galaxies located within $11 \mathrm{Mpc}$. The results of our previous observations are reported in a series of 9 papers references to which can be found in the last paper of this series [1].

Mass sky surveys made at optical wavelengths and in the HI $21 \mathrm{~cm}$ line gave rise to a rapid increase of the number of galaxies in the LV. Their number in the "Updated Nearby Galaxy Catalog" (UNGC) [2] reached 869, and more than $80 \%$ of the sample are made up of dwarf galaxies with stellar masses $\log \left(M^{*} / M_{\odot}\right)<9.0$. As of mid-2018 the LV galaxy database [3] included 1153 objects $^{1}$. $\mathrm{H} \alpha$-images for a total of more than 300 nearby galaxies were acquired with the 6 -m telescope of the Special Astrophysical Observatory of the Russian Academy of Sciences within the framework of our $\mathrm{H} \alpha$-survey program. Our survey, combined with other similar surveys [4-6], makes up a more than 500-object sample of LV galaxies with measured $\mathrm{H} \alpha$. Most of these galaxies have their ultraviolet fluxes measured with GALEX [7] space telescope, making it possible to estimate the star-formation rates in galaxies on the time scale of about $100 \mathrm{Myr}$, which is about one order of magnitude longer than the time interval gauged by star-formation rate estimates based on $\mathrm{H} \alpha$ emission. A comparison of the two star-formation rates, $\mathrm{SFR}_{\mathrm{H} \alpha}$ and $\mathrm{SFR}_{\mathrm{FUV}}$, makes it possible to reveal starburst and star-formation rate deceleration on 10-100 Myr long time intervals.

\footnotetext{
Based on observations with the 6-m telescope of the Special Astrophysical Observatory of the Russian Academy of Sciences.

** Electronic address: skai@sao.ru

${ }^{1}$ Various data about these objects are available at http://www. sao.ru/lv/lvgdb
} 
Below we present the $\mathrm{H} \alpha$-images and the integrated $\mathrm{H} \alpha$ fluxes for $66 \mathrm{LV}$ objects. Almost all these objects are dwarf galaxies except for three late-type spirals: IC 2233, UGC 4704, and NGC 3432, and six globular clusters at the distant (farther than $100 \mathrm{kpc}$ ) periphery of the Andromeda galaxy (M31). Only six galaxies of this list had their $\mathrm{H} \alpha$-fluxes measured earlier, and in three cases (JKB 83, Grapes and NGC 6503-d1) we found greater than one order of magnitude errors in previous $\mathrm{H} \alpha$-flux measurements.

\section{OBSERVATIONS AND DATA REDUCTION}

Observations of nearby dwarf galaxies were made on the 6-m telescope of the Special Astrophysical Observatory of the Russian Academy of Sciences in 2015-2018 using SCORPIO focal reducer [8]. Galaxy images were registered with $2048 \times 2048$ CCD providing a field of view of $6 ! 1 \times 6 ! 1$ and a resolution of 0 .'18 $\mathrm{pix}^{-1}$. H $\alpha$ galaxy images and galaxy images in the neighboring continuum were obtained using a narrow-band $\mathrm{H} \alpha$ interference filter with a width of $\Delta \lambda=74 \AA$ and effective wavelength of $\lambda_{\text {eff }}=6555 \AA$, and two intermediate-band filters SED 607 with $\Delta \lambda=167 \AA, \lambda_{\text {eff }}=6063 \AA$ and SED 707 with $\Delta \lambda=207 \AA, \lambda_{\text {eff }}=7063 \AA$. Typical exposures were $2 \times 600$ and $2 \times 300 \mathrm{~s}$ in the $\mathrm{H} \alpha$ line and continuum, respectively. Because of the small range of radial velocities of nearby galaxies we exposured their $\mathrm{H} \alpha$ images with the same filter. The radial velocities of the objects studied lie in the interval from $-333 \mathrm{~km} \mathrm{~s}^{-1}$ for PAndAS-01 to $844 \mathrm{~km} \mathrm{~s}^{-1}$ for UGCA 298.

We reduced observational data in a standard way using MIDAS package. After subtraction of the electronic bias frame we performed flat field correction based on with flat field frames obtained by taking sky images during twilight. We then removed cosmic-ray hits, aligned the frames, and subtracted the continuum normalized by the images of $5-15$ stars located in the frame. We calibrated the measured integrated $\mathrm{H} \alpha$ fluxes of the galaxies by images of stellar spectrophorometric standards acquired on the same night. Internal $\mathrm{H} \alpha$-flux measurement errors were typically of about $15 \%$. Variations of atmospheric conditions was the main source of these errors. The resulting $\mathrm{H} \alpha$ fluxes also included the contribution from the [N II] doublet emission lines, which was small in the case of dwarf galaxies and we ignored it. Only in the case of three late-type spiral galaxies we corrected the measured fluxes for the contribution of the [N II] doublet in accordance with [9].

\section{RESULTS OF OBSERVATIONS}

We present the images of the observed galaxies in the form of an atlas shown in Fig. 1. The leftand right-side images of each galaxy represent the sum and difference of the $\mathrm{H} \alpha$ and continuum images. The angular size of the images is $4^{\prime} \times 4^{\prime}$, the arrows show the direction toward North and East. In some frames interference pattern can be seen, which is due to imperfect account of the division by flat field. The bright stars and objects with anomalous color of the wave in the right-hand images as residual "stumps". We corrected the measured integrated H $\alpha$-flux of the 

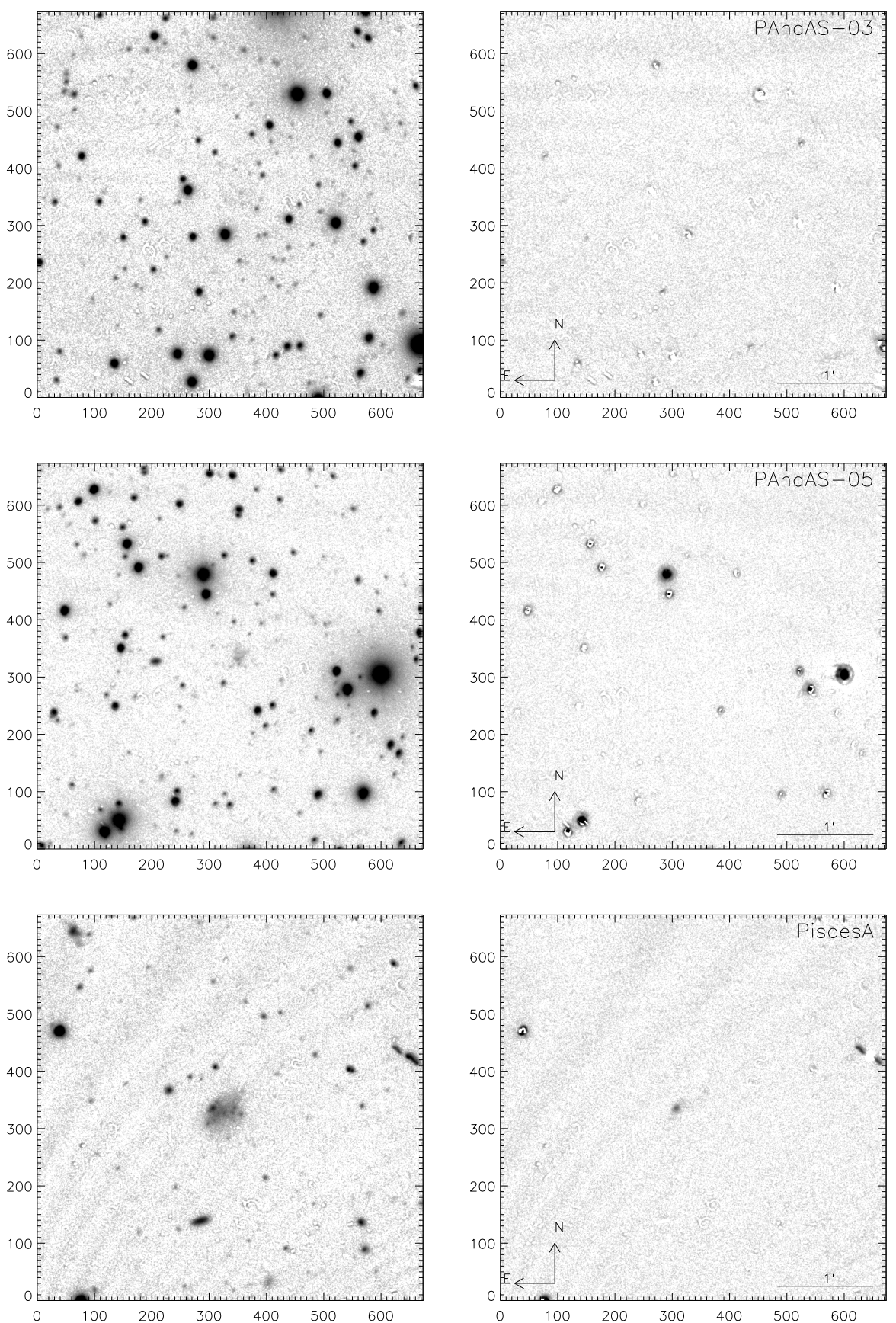

Figure 1. Atlas of LV object images. The left-side images in each pair are coadded $\mathrm{H} \alpha$ and continuum exposures, and the right-side images show the differences of these exposures. The angular scale and orientation are indicated in the right-hand images. Here we show only the first page of the image. The complete image files are available at https://www.sao.ru/hq/leac/recent_results/Halpha_atlas_66.pdf. 
Table 1. General parameters of observed galaxies

\begin{tabular}{|c|c|c|c|c|c|c|c|c|}
\hline Name & RA (2000) Dec & $\begin{array}{l}B_{T} \\
\mathrm{mag}\end{array}$ & $\mathrm{T}$ & \begin{tabular}{r|} 
\\
$\mathrm{Mpc}$ \\
\end{tabular} & $\log F_{H \alpha}$ & $\begin{array}{r}\log S F R_{H \alpha}, \\
{\left[M_{\odot} \mathrm{yr}^{-1}\right]} \\
\end{array}$ & $\begin{array}{c}\log S F R_{F U V}, \\
{\left[M_{\odot} \mathrm{yr}^{-1}\right]}\end{array}$ & $\begin{array}{r}\log s S F R_{H \alpha} \\
{\left[\mathrm{yr}^{-1}\right]}\end{array}$ \\
\hline$\overline{(1)}$ & $(2)$ & $(3)$ & (4) & (5) & (6) & (7) & $(8)$ & (9) \\
\hline PAndAS-03 & $000356.4+405319$ & 21.3 & -3 & 0.78 & $<-15.10$ & $<-6.26$ & $<-6.35$ & $<-10.64$ \\
\hline PAndAS-05 & $000524.1+435535$ & 20.3 & -3 & 0.78 & $<-15.30$ & $<-6.46$ & $<-6.38$ & $<-11.21$ \\
\hline PiscesA & $001446.0+104847$ & 18.5 & 10 & 5.65 & -14.25 & -3.67 & - & -10.20 \\
\hline JKB129 & $002041.4+083701$ & 17.4 & 10 & 7.50 & -13.87 & -3.03 & -2.69 & -10.27 \\
\hline PAndAS-50 & $010150.6+481819$ & 19.3 & -3 & 0.78 & $<-15.12$ & $<-6.21$ & $<-6.10$ & $<-11.49$ \\
\hline PiscesB & $011911.7+110718$ & 17.8 & 10 & 8.91 & -13.94 & -3.02 & -2.80 & -10.13 \\
\hline PAndAS-55 & $011920.4+460312$ & 20.0 & -3 & 0.78 & $<-15.16$ & $<-6.33$ & $<-6.40$ & $<-11.20$ \\
\hline AGC112454 & $013259.7+142224$ & 17.5 & 9 & 10.20 & -13.39 & -2.35 & - & -9.70 \\
\hline AGC114027 & $013441.8+143840$ & 18.8 & 10 & 9.90 & -13.87 & -2.85 & - & -9.66 \\
\hline AGC112503 & $013800.3+145858$ & 18.0 & 9 & 10.20 & -13.61 & -2.57 & - & -9.72 \\
\hline N672dwB & $014711.1+274100$ & 21.0 & 10 & 7.20 & $<-15.24$ & $<-4.48$ & $<-4.46$ & $<-10.17$ \\
\hline N672dwA & $014719.1+271516$ & 19.8 & 10 & 7.20 & $<-15.21$ & $<-4.44$ & -4.48 & $<-10.62$ \\
\hline Triangulum-II & $021317.4+361042$ & 16.5 & -2 & 0.03 & $<-15.17$ & $<-9.16$ & - & $<-12.61$ \\
\hline AGC123352 & $024839.2+231626$ & 19.2 & 10 & 8.27 & -14.01 & -3.00 & -3.28 & -9.77 \\
\hline AGC124056 & $025736.4+234721$ & 19.0 & 10 & 7.37 & $<-15.20$ & $<-4.36$ & $<-4.23$ & $<-10.98$ \\
\hline N1156dw1 & $030018.2+251456$ & 19.6 & 10 & 7.80 & $<-15.11$ & $<-4.10$ & $<-3.76$ & $<-10.75$ \\
\hline N1156dw2 & $030028.0+251817$ & 20.0 & 10 & 7.80 & $<-15.25$ & $<-4.23$ & -3.21 & $<-10.74$ \\
\hline GALFA-Dw 4 & $054544.8+104616$ & 17.8 & 10 & 7.22 & -13.24 & -2.05 & - & -9.81 \\
\hline MADCASH & $074238.9+652502$ & 20.5 & -2 & 3.39 & $<-15.26$ & $<-5.19$ & $<-5.26$ & $<-11.05$ \\
\hline IC2233 & $081358.9+454432$ & 13.1 & 7 & 12.20 & -12.11 & -0.75 & -0.26 & -9.97 \\
\hline LV J0831+4104 & $083141.2+410454$ & 17.7 & 9 & 7.90 & -15.25 & -4.44 & -3.26 & -11.46 \\
\hline LV J0843+4025 & $084338.0+402547$ & 17.9 & 9 & 7.80 & -13.89 & -3.10 & -2.99 & -10.02 \\
\hline N2683dw2 & $085420.5+331458$ & 19.6 & -2 & 9.82 & $<-15.28$ & $<-4.29$ & $<-4.34$ & $<-11.43$ \\
\hline UGC04704 & $085900.3+391236$ & 15.0 & 8 & 15.20 & -12.84 & -1.38 & -0.89 & -10.31 \\
\hline AGC198507 & $091525.8+252510$ & 18.6 & 9 & 8.90 & $-15.01:$ & -4.10 : & -3.20 & $-10.87:$ \\
\hline JKB83 & $095549.6+691957$ & 19.7 & 10 & 3.70 & -13.67 & -3.49 & -4.24 & -9.14 \\
\hline PGC30114 & $101843.0+460244$ & 15.7 & 8 & 8.05 & -12.54 & -1.74 & -1.88 & -9.63 \\
\hline UGC05571 & $101942.4+520356$ & 16.5 & 9 & 8.24 & -13.39 & -2.57 & -2.24 & -10.06 \\
\hline LV J1028+4240 & $102833.0+424008$ & 17.4 & 10 & 7.55 & -14.13 & -3.38 & -2.67 & -10.44 \\
\hline PGC2277751 & $103512.1+461412$ & 17.4 & 9 & 7.25 & -14.57 & -3.85 & -3.15 & -10.90 \\
\hline N3344dw1 & $104244.0+250130$ & 20.0 & 10 & 9.82 & $<-15.30$ & $<-4.31$ & $<-4.33$ & $<-10.60$ \\
\hline Willman1 & $104921.0+510300$ & 16.0 & -2 & 0.04 & $<-15.22$ & $<-9.03$ & -8.51 & $<-12.75$ \\
\hline NGC3432 & $105231.1+363708$ & 11.7 & 8 & 9.20 & -11.24 & -0.15 & -0.00 & -9.79 \\
\hline LV J1052+3639 & $105240.8+363954$ & 15.8 & 10 & 9.20 & -12.86 & -1.94 & -2.01 & -9.82 \\
\hline HS1053+3624 & $105640.4+360828$ & 17.8 & 10 & 9.20 & -13.51 & -2.58 & -2.54 & -9.69 \\
\hline VV747 & $105747.0+361539$ & 15.5 & 10 & 9.20 & -12.63 & -1.70 & -1.85 & -9.72 \\
\hline PGC034671 & $111948.6+554322$ & 16.5 & 9 & 9.90 & -14.22 & -3.24 & -2.81 & -10.91 \\
\hline UGC06757 & $114659.1+612006$ & 16.3 & 10 & 4.61 & -14.12 & -3.78 & -3.02 & -10.90 \\
\hline
\end{tabular}


Table 1.

\begin{tabular}{|c|c|c|c|c|c|c|c|c|}
\hline Name & RA (2000) Dec & $\begin{array}{l}B_{T} \\
\mathrm{mag} \\
\end{array}$ & $\mathrm{T}$ & $\begin{array}{r}D, \\
\mathrm{Mpc} \\
\end{array}$ & $\log F_{H \alpha}$ & $\begin{array}{r}\log S F R_{H \alpha}, \\
{\left[M_{\odot} \mathrm{yr}^{-1}\right]} \\
\end{array}$ & $\begin{array}{c}\log \left[S F R_{F U V}\right. \\
{\left[M_{\odot} \mathrm{yr}^{-1}\right]}\end{array}$ & $\begin{array}{r}\log s S F R_{H \alpha} \\
{\left[\mathrm{yr}^{-1}\right]} \\
\end{array}$ \\
\hline$(1)$ & $(2)$ & (3) & (4) & $(5)$ & (6) & $(7)$ & (8) & (9) \\
\hline Grapes & $115205.6+544732$ & 18.5 & 10 & 5.58 & -14.63 & -4.15 & - & -10.51 \\
\hline PGC3401153 & $115352.4+512938$ & 17.5 & 9 & 7.79 & -14.63 & -3.85 & -3.06 & -10.90 \\
\hline MAPS1206+31 & $120634.5+312033$ & 15.8 & 8 & 7.43 & -13.78 & -3.04 & -2.97 & -10.85 \\
\hline UGC07320 & $121728.5+444841$ & 15.4 & 10 & 9.20 & -13.87 & -2.95 & -2.61 & -10.99 \\
\hline KK135 & $121934.7+580234$ & 18.1 & 10 & 4.46 & -13.84 & -3.54 & -3.36 & -9.86 \\
\hline PGC5059199 & $122409.9+261352$ & 19.8 & 10 & 3.37 & -14.59 & -4.53 & - & -9.97 \\
\hline AGC724906 & $123056.0+263040$ & 17.4 & 10 & 7.55 & -15.17 & -4.41 & -3.18 & -11.50 \\
\hline DDO133 & $123253.0+313221$ & 13.5 & 10 & 4.88 & -12.36 & -1.98 & -1.73 & -10.22 \\
\hline PGC041749 & $123352.7+393733$ & 16.5 & 9 & 8.24 & -13.46 & -2.64 & -2.11 & -10.15 \\
\hline UGC7751 & $123511.8+410339$ & 16.5 & 9 & 7.90 & -13.89 & -3.09 & -2.43 & -10.58 \\
\hline AGC749241 & $124001.7+261919$ & 18.3 & 10 & 5.62 & -15.28 & -4.79 & -3.54 & -11.25 \\
\hline KDG178 & $124010.0+323931$ & 17.1 & 10 & 7.30 & -14.23 & -3.51 & -2.64 & -10.67 \\
\hline NGC4656UV & $124415.7+321700$ & 13.0 & 10 & 5.40 & -13.11 & -2.66 & -1.11 & -11.20 \\
\hline UGCA298 & $124655.4+263351$ & 15.3 & 9 & 11.00 & -12.73 & -1.66 & -2.23 & -10.09 \\
\hline KKH82 & $131258.7+414712$ & 16.4 & 10 & 8.06 & -15.07 & -4.27 & -3.55 & -11.80 \\
\hline PGC2229803 & $132753.1+434855$ & 16.2 & 9 & 7.24 & -13.90 & -3.19 : & -2.89 & $-10.69:$ \\
\hline LV J1328+4937 & $132831.2+493738$ & 17.3 & 10 & 8.40 & -13.79 & -2.95 & -2.54 & -10.15 \\
\hline AGC238890 & $133230.3+250725$ & 16.6 & 9 & 6.80 & -14.60 & -3.94 & - & -11.24 \\
\hline UGC08647 & $133948.0+311725$ & 16.3 & 8 & 8.60 & -13.30 & -2.44 & -2.43 & -10.18 \\
\hline LV J1342+4840 & $134220.1+484057$ & 16.3 & 9 & 8.40 & -14.21 & -3.37 & - & -10.97 \\
\hline dw1343+58 & $134307.0+581340$ & 15.7 & 9 & 6.95 & -13.84 & -3.17 & -2.56 & -10.82 \\
\hline PGC2448110 & $140457.8+534129$ & 17.3 & 10 & 5.38 & -12.59 & -2.14 & -3.05 & -8.94 \\
\hline M101-df2 & $140837.5+541931$ & 19.8 & -2 & 6.87 & $<-15.26$ & $<-4.60$ & - & $<-11.34$ \\
\hline UGC09540 & $144852.0+344243$ & 16.8 & 10 & 9.30 & -12.99 & -2.05 & -2.10 & -9.57 \\
\hline NGC6503-d1 & $175248.4+700814$ & 18.6 & 10 & 6.25 & -15.33 & -4.73 & -4.09 & -11.17 \\
\hline Pegasus III & $222422.6+052512$ & 18.7 & -2 & 0.21 & $<-15.26$ & $<-7.52$ & - & $<-11.83$ \\
\hline PAndAS-01 & $235712.0+433308$ & 17.9 & -3 & 0.78 & $<-15.20$ & $<-6.35$ & $<-6.33$ & $<-12.09$ \\
\hline PAndAS-02 & $235755.6+414649$ & 18.7 & -3 & 0.78 & $<-15.20$ & $<-6.34$ & $<-6.28$ & $<-11.79$ \\
\hline
\end{tabular}

galaxy, $F\left(\mathrm{H} \alpha\right.$ ) (in the units of $\mathrm{erg} \mathrm{cm}^{-2} \mathrm{~s}^{-1}$ ), for Galactic extinction in accordance with [10]. The absorption-corrected value, $F_{c}(\mathrm{H} \alpha)$, served for estimating the integrated star-formation rate in the galaxy [11] in the units of $\left(M_{\odot} \mathrm{yr}^{-1}\right)$ :

$$
\log (S F R)=\log F_{c}(\mathrm{H} \alpha)+2 \log D+8.98
$$

where $D$ is the distance to the galaxy in Mpc. Here we ignore the contribution from the flux of the [N II] emission doublet and the internal absorption in the Galaxy, because both these effects are small for dwarf galaxies [12], which make up a for the majority of observed objects. The only exceptions were three spiral galaxies IC 2233, UGC 4704 and NGC 3432, seen edge-on, for which 
we adopted internal extinction in the $\mathrm{H} \alpha$-line equal to $0.73,0 \mathrm{~m} 40$, and 0.77 , respectively, in accordance with [13].

The list of general parameters of observed galaxies is presented in Table 1. Its columns give: (1) - the name of the galaxy; (2) - J2000.0 equatorial coordinates; (3)-integrated B-band magnitude; (4)-morphological type according to UNGC [2]; (5) - distance in Mpc according to [2]; (6) - the logarithm of our measured $\mathrm{H} \alpha$ flux; (7) - the logarithm of the integrated SFR according to the above formula; (8) - the integrated SFR of the galaxy

$$
\log \left(S F R_{\mathrm{FUV}}\right)=\log F_{c}(\mathrm{FUV})+2 \log D-6.78
$$

determined from its far ultraviolet flux $\left(\lambda_{e}=1539 \AA\right.$, FWHM $\left.=269 \AA\right)$ measured with GALEX satellite [7] corrected for internal extinction in the Galaxy; (9)-specific SFR (sSFR) normalized to unit stellar mass adopted from the UNGC catalog [2]. Several H $\alpha$-flux estimates obtained from observations made through cirrus clouds are marked by colons.

\section{SOME FEATURES OF OBSERVED OBJECTS}

PAndAS-03, PAndAS-05, PAndAS-50, PAndAS-55, PAndAS-01, and PAndAS-02. Global clusters at the distant periphery of our neighboring M31 galaxy. No signs of star formation have been detected in these objects wither in $\mathrm{H} \alpha$-images or in the FUV-flux.

Pisces A, Pisces B. Two isolated gas-rich dwarf galaxies discovered by Tollerud et al. [14]. The tip of the red giant branch (TRGB) distances to these galaxies were measured with the HST [15].

AGC 112454. Gas-rich dwarf galaxy from ALFALFA H I-survey [16], likely satellite of the spiral galaxy NGC628. Emission regions in AGC 112454 have the form of two touching rings.

N672A, N 672B. Two likely dwarf satellites of the NGC 672 galaxy found on long-exposure images taken with a small amateur telescope [17].

Triangulum-II, Wilman 1, Pegasus-III. Dwarf spheroidal satellites of the Milky Way resolved into stars [18-20].

$A G C$ 124056. Gas-rich low surface brightness dwarf galaxy from ALFALFA survey. A bright star is located north of this galaxy.

N1156dw 1, N1156dw2. Two likely dwarf satellites of the NGC 1156 galaxy [17].

GALFA-Dw4. Irregular dwarf galaxy from the catalog of compact H I-clouds [21].

MADCASH J0742+65. Ultra-dwarf spheroidal galaxy, a satellite of the spiral galaxy NGC 2403 [22].

IC 2233. This edge-on Sd galaxy has a radial velocity and $\mathrm{H}$ I-line width of $V_{\mathrm{LG}}=586 \mathrm{~km} \mathrm{~s}^{-1}$ and $W_{50}=178 \mathrm{~km} \mathrm{~s}^{-1}$, respectively. Based on the Tully-Fisher [23] relation, its distance is $12.2 \mathrm{Mpc}$, which is somewhat greater than the given limit of the Local Volume (11 Mpc). Our estimate $\log \left(S F R_{\mathrm{H} \alpha}\right)=-0.75$ agrees well with the estimate -0.71 , obtained earlier by Kennicutt et al. [6]. 
N2683dw2. Dwarf spheroidal galaxy discovered on an image obtained with an amateur telescope [17], a likely satellite of the spiral galaxy NGC 2683.

$U G C$ 4704. Gas-rich Sdm-type galaxy seen almost exactly edge-on. Its distance estimated by the Tully-Fisher relation is $D=15.2 \mathrm{Mpc}$. With its velocity $V_{\mathrm{LG}}=584 \mathrm{~km} \mathrm{~s}^{-1}$, the galaxy is located in the region of Local Velocity Anomaly [24]. Our SFR estimate, $\log \left(S F R_{\mathrm{H} \alpha}\right)=-1.38$, agrees well with the -1.42 value obtained by Kennicutt et al. [6].

$J K B$ 83. Intergalactic HII-region in the M 81 group. James et al. [25] report an order-ofmagnitude higher $\mathrm{H} \alpha$-flux than our measured value. Our repeated images of JKB 83 made with the 6-m telescope confirmed its small $\mathrm{H} \alpha$ flux.

$N 3344 d w 1$. A low surface brightness dwarf galaxy in the vicinity of the spiral galaxy NGC 3344 . NGC 3432=Arp 206=VV11. An Sdm galaxy with distorted structure. Kennicut et al. [6] report a 0.10 dex lower $\mathrm{H} \alpha$ flux than our estimate.

$J 1052+3639$. Dwarf satellite in contact with NGC 3432. Shows up conspicuously in the GALEX FUV-band.

HS 1053+3624 and VV747. These are two likely satellites of NGC 3432.

Grapes. An isolated dwarf galaxy with a radial velocity of $V_{\mathrm{LG}}=408 \mathrm{~km} \mathrm{~s}^{-1}$ [26]. Its distance is estimated from its $V_{\mathrm{LG}}$ adopting a Hubble constant of $H_{0}=73 \mathrm{~km} \mathrm{~s}^{-1} \mathrm{Mpc}^{-1}$. Our measured $\mathrm{H} \alpha$ flux of this galaxy is 1.07 dex higher than reported in [26].

MAPS 1206+31. Blueish emission galaxy with a small high-contrast nucleus, which breaks up into several compact nodes on the $\mathrm{H} \alpha$ image.

$K K$ 135. An isolated dIr galaxy with a radial velocity of $V_{\mathrm{LG}}=326 \mathrm{~km} \mathrm{~s}^{-1}$, implying a kinematic distance of $4.46 \mathrm{Mpc}$. It is a possible peripheral member of the M 81 group. On the H $\alpha$ image KK 135 has the form of an open ring with one emission-line condensation.

$P G C 5059199=A G C$ 749235. A small blue gas-rich dwarf galaxy from the ALFALFA survey [16] with a radial velocity of $V_{\mathrm{LG}}=246 \mathrm{~km} \mathrm{~s}^{-1}$ and the $\mathrm{H}$ I-line width of $W_{50}=98 \mathrm{~km} \mathrm{~s}^{-1}$. Its structure is inconsistent with the kinematic distance of $3.37 \mathrm{Mpc}$. Possibly a member of the peculiar Coma I group at the distance of $16 \mathrm{Mpc}$.

DDO 133. An Im galaxy at a distance of $4.88 \mathrm{Mpc}$ determined from the TRGB [27]. The galaxy contains more than a dozen compact and ring-shaped H II-regions.

$N G C 4656 U V$. A dwarf satellite of NGC 4656, in contact with the main galaxy and located northeast from it. The galaxy has low surface brightness in the optical continuum and $\mathrm{H} \alpha$ line, but shows up contrastively in the FUV-band [28].

UGCA 298=Mrk 1335. A blue compact dwarf galaxy at the boundary of the LV (11.0 Mpc) with smooth structure and bright emission-line nucleus. Judging by its $S F R_{\mathrm{H} \alpha} / S F R_{\mathrm{FUV}} \sim 2.7$ ratio, Mrk 1335 undergoes the stage of flare activity.

KKH 82 and PGC 2229803, LV J1328+4937 and LV J1342+4840. Likely satellites of the bright M 51 pair of galaxies.

$d w 1343+58$. Given its radial velocity, this dwarf galaxy is a satellite of the giant spiral galaxy 

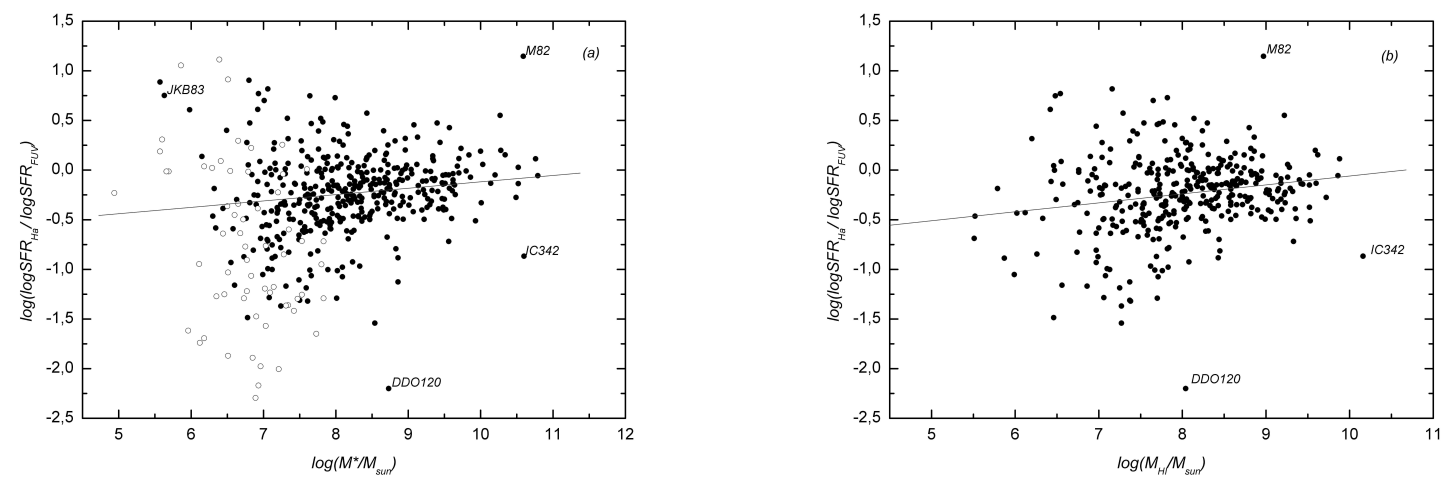

Figure 2. The ratio or the SFR determined from $\mathrm{H} \alpha$ and FUV fluxes as a function of stellar mass (a) and hydrogen mass of the galaxy (b).

$\mathrm{M} 101\left(V_{\mathrm{LG}}=365 \mathrm{~km} \mathrm{~s}^{-1}\right)$.

PGC 2448110. Compact H II region at the periphery of the Sm galaxy NGC 5474-a satellite of M 101. In PanSTARRS survey images [29] compact nodes can be seen inside this H II region. The $S F R_{\mathrm{H} \alpha} / S F R_{\mathrm{FUV}}=8.1$ ratio is indicative of violent starburst phase in his object.

M 101-df2. A new dwarf spheroidal satellite of the M 101 galaxy. Its TRGB distance, 6.87 Mpc [30], confirms the membership of this dwarf in the M 101 group.

$U G C 09540=K K R$ 13. An isolated gas-rich dIr galaxy. Its distance, $9.30 \mathrm{Mpc}$, was estimated by the baryonic Tully-Fisher relation [31].

KK 242 = NGC6503-D1. Low surface brightness dwarf galaxy found by Karachentseva and Karachentsev [32] in the vicinity of the spiral galaxy NGC6503. Koda et al. [33] independently described it as NGC 6503-D1 and reported a 12 times overestimated $\mathrm{H} \alpha$ flux. According to [34], the radial velocity of KK 242 is $V_{\text {hel }}=426 \pm 6 \mathrm{~km} \mathrm{~s}^{-1}$, its H I flux and the line width are $F(\mathrm{HI})=2.03 \mathrm{Jy} \mathrm{km}^{-1} \mathrm{~s}^{-1}$ and $W_{50}=100 \mathrm{~km} \mathrm{~s}^{-1}$, respectively. These parameters, which are unusual for a dwarf galaxy, have to be independently verified.

\section{RESULTS AND DISCUSSION}

We combined the above star-formation rate estimates in LV galaxies with similar data for other galaxies located within $11 \mathrm{Mpc}$. It was already pointed out in Ricciardelli et al. [35] and Karachentsev et al. [36] that the SFR in late-type galaxies depends only slightly on the neighborhood. The trend of the decrease of SFR from isolated galaxies to group members barely exceeds the dispersion of this quantity and is most manifested among the least massive dwarf galaxies. In other words, SFR in galaxies is mostly determined by their individual properties rather than by the tidal effects produced by neighbours.

Fig. 2 shows the distribution of the ratio of SFR estimates determined from $\mathrm{H} \alpha$ and FUV fluxes 

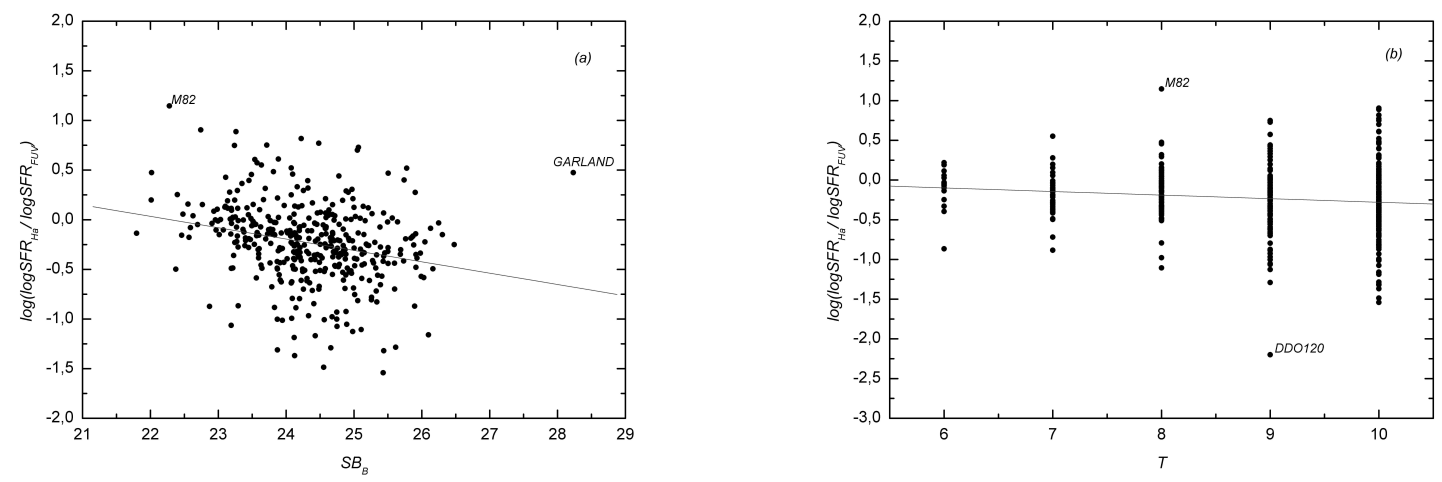

Figure 3. Ratio of the SFR estimates determined from $\mathrm{H} \alpha$ and FUV fluxes as a function of average surface brightness in magnitudes per square arcsecond $\left({ }^{\mathrm{m}} / \square^{\prime \prime}\right)(\mathrm{a})$ and morphological type $\mathrm{T}(\mathrm{b})$.

for $506 \mathrm{LV}$ galaxies. The left- and right-side panels show how this ratio depends on the stellar and hydrogen masses of galaxies, respectively. The sample includes only late-type galaxies: $\mathrm{T}$ $=10(\mathrm{Ir}), 9(\mathrm{Im}, \mathrm{BCD}), 8(\mathrm{Sdm}), 7(\mathrm{Sd})$, and $6(\mathrm{Scd})$, because elliptical and lenticular galaxies as well as spiral galaxies with massive bulges $(\mathrm{T} \leq 5)$ have en essentially different star-formation history. Galaxies with measured FUV fluxes but with only an upper $\mathrm{H} \alpha$ flux limit are indicated in Fig. 2 by open circles and we did not took them into account when computing the statistics of the $S F R_{\mathrm{H} \alpha} / S F R_{\mathrm{FUV}}$ ratio. It follows from the data reported here that the $S F R_{\mathrm{H} \alpha} / S F R_{\mathrm{FUV}}$ shows a tendency to decrease toward low-mass galaxies and the dispersion of this ratio increases significantly from spiral to dwarf galaxies. Such features of the diagram are due to the burst nature of star formation in dwarf galaxies, where the amplitude of starbursts on a (10-100) Myr time scale increases with the decreasing stellar and hydrogen mass of the galaxy [12, 37-41].

Particularly striking examples are the M 82 and Mrk 475 galaxies in the starburst phase and the DDO 120 galaxy in the post-starburst quiescent phase with their $S F R_{\mathrm{H} \alpha} / S F R_{\mathrm{FUV}}$ ratios differing by more than three orders of magnitude.

Meurer et al. [42] and Audcent-Ross et al.[43] pointed out that the burstlike nature of star formation in low-mass galaxies shows up in their average surface brightness. The left-side panel in Fig. 3 shows the dependence of the $S F R_{\mathrm{H} \alpha} / S F R_{\mathrm{FUV}}$ ratio on the average B-band surface brightness of galaxies within the Holmberg radius. As is evident from the regression line, the average $S F R_{\mathrm{H} \alpha} / S F R_{\mathrm{FUV}}$ ratios of BCD galaxies $\left(S B \sim 22^{\mathrm{m}} / \square^{\prime \prime}\right)$ differ from those of low surface brightness dwarfs $\left(S B \sim 26^{\mathrm{m}} / \square^{\prime \prime}\right)$ by about a factor of 0.5 dex.

The right-side panel in Fig. 3 reproduces the distribution of the $S F R_{\mathrm{H} \alpha} / S F R_{\mathrm{FUV}}$ ratio for galaxies of different morphological types. Table 2 lists the average ratio and its standard deviation along with the average hydrogen mass and its standard deviation for galaxies of different types. The data in the table indicate that the average $S F R_{\mathrm{H} \alpha} / S F R_{\mathrm{FUV}}$ ratio decreases smoothly from spiral to irregular dwarf galaxies, and the dispersion of this ratio increases toward dwarf galaxies 
Table 2. Average integrated parameters and the standard deviations for galaxies of late morphological types with SFR estimates

\begin{tabular}{r|r|c|c|c|c|c|c}
\hline $\mathrm{T}$ & $\mathrm{N}$ & $\left\langle\log M_{*}\right\rangle$ & $\mathrm{SD}$ & $\left\langle\log M_{\mathrm{HI}}\right\rangle$ & $\mathrm{SD}$ & $\left\langle\log \left(S F R_{\mathrm{H} \alpha} / S F R_{\mathrm{FUV}}\right)\right\rangle$ & $\mathrm{SD}$ \\
\hline 6 & 23 & $9.98 \pm 0.10$ & 0.48 & $9.23 \pm 0.08$ & 0.39 & $-0.11 \pm 0.06$ & 0.26 \\
7 & 40 & $9.25 \pm 0.09$ & 0.55 & $8.86 \pm 0.07$ & 0.45 & $-0.19 \pm 0.04$ & 0.26 \\
8 & 96 & $8.88 \pm 0.06$ & 0.58 & $8.53 \pm 0.05$ & 0.49 & $-0.15 \pm 0.03$ & 0.31 \\
9 & 118 & $8.12 \pm 0.06$ & 0.64 & $7.72 \pm 0.06$ & 0.69 & $-0.25 \pm 0.04$ & 0.45 \\
10 & 229 & $7.59 \pm 0.04$ & 0.64 & $7.56 \pm 0.04$ & 0.64 & $-0.28 \pm 0.03$ & 0.46 \\
\hline
\end{tabular}

thereby confirming the idea about the burstlike nature of star-formation activity in low-mass objects. Taking into account the LV galaxies for which only the upper $\mathrm{H} \alpha$ flux limit is determined makes this dependence even steeper and more significant.

The LV contains more than 100 galaxies with measured $\mathrm{H} \alpha$ fluxes, which are located outside the coverage zone of the GALEX UV survey. The data from Table 2 can be used to obtain an unbiased estimate of their FUV flux from known $\mathrm{H} \alpha$ flux depending on the morphology of the galaxy.

The integrated SFR is closely correlated with the luminosity or mass of the galaxy. Therefore the star-formation process is usually characterized by the so-called specific star-formation rate, $s S F R=S F R / M_{*}$, i.e., SFR per unit stellar mass. Along with it, the "star-formation efficiency" parameter, $S F E=S F R / M_{\mathrm{HI}}$, is used, which indicates how quickly the available gas resource in the galaxy will be exhausted.

Given that both the stellar and gas masses of the galaxy vary in the process of its evolution, it is reasonable to introduce yet another parameter into the description of evolution without external influence (the "closed box" model) - specific star-formation rate per unit baryonic mass of the galaxy, $b S F R=S F R /\left(M_{*}+1.85 M_{\mathrm{HI}}\right)$. Here the factor 1.85 takes into account the contribution of helium and molecular hydrogen to the total mass of gas [44].

Fig. 4 shows the distribution of the following parameters of LV galaxies: $s S F R, S F E$, and $b S F R$. In each case SFR is determined from measured $\mathrm{H} \alpha$ flux. The distributions for galaxies with the upper $\mathrm{H} \alpha$ flux levels are shown with unhatched bars. The last plot in the figure corresponds to $b S F R$ determined from FUV fluxes. An analysis of these histograms leads us to the following conclusions.

(a) The distributions of SFRs of late-type galaxies $(T=10-6)$ normalized to unit stellar mass, unit hydrogen mass, or unit total baryonic mass are asymmetric with an excess of galaxies at low SFR. The inclusion of galaxies with FUV or $\mathrm{H} \alpha$ fluxes below the detection limit (about $10 \%$ of the entire sample) makes the observed asymmetry even stronger.

(b) The median values of the distributions:

$s S F R_{\mathrm{H} \alpha}=-10.31 \mathrm{dex}, b S F R_{\mathrm{H} \alpha}=-10.64 \mathrm{dex}$ and $b S F R_{\mathrm{FUV}}=-10.52 \mathrm{dex}$

are close to each other and are a factor of 1.5-3 smaller than the expansion rate of the Universe, 

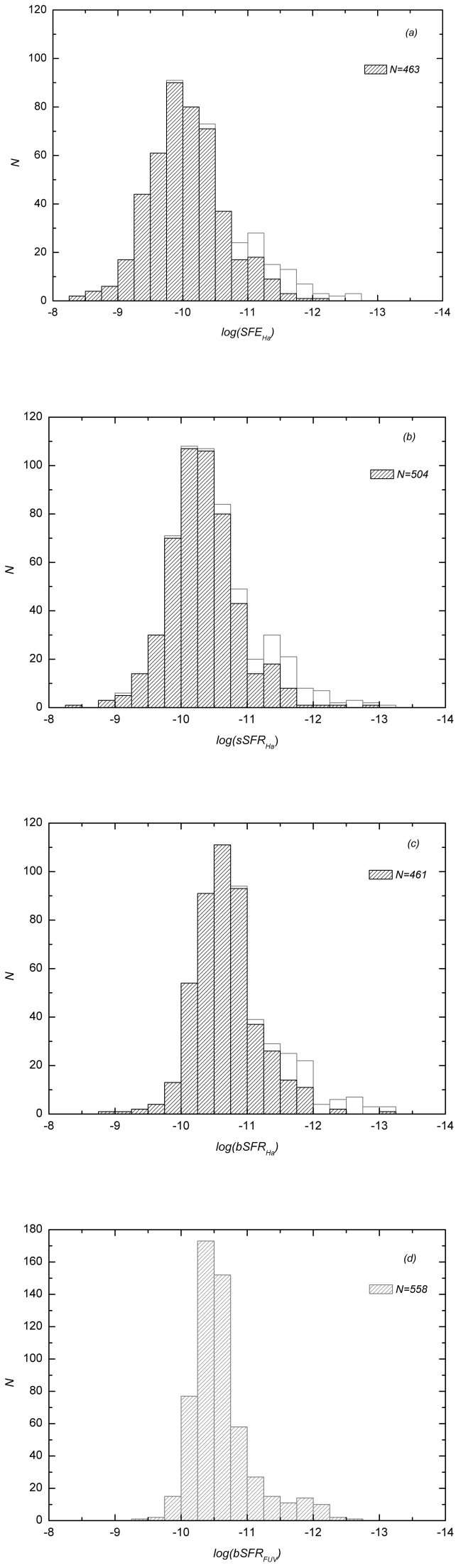

Figure 4. Distribution of (a) $S F E$; (b) $s S F R$ determined from $\mathrm{H} \alpha$ flux; (c) $b S F R$ determined from $\mathrm{H} \alpha$ flux; (d) $b S F R$ determined from FUV measurements for LV galaxies. The horizontal scale is in the units of $\left[\mathrm{yr}^{-1}\right]$ in all plots. 
$H_{0}=-10.14$. The observed SFR in a typical late-type LV galaxy is quite sufficient for reproducing most of its observed stellar mass during cosmological time $H_{0}^{-1} \simeq 13.7$ Gyr. In other words, irregular dwarf galaxies and disks of spiral galaxies may be evolving in "glowing" mode without violent starburst phase at $z \geq 2$ typical of early-type galaxies. Note that reserves of gas in type $\mathrm{T}=10-6$ galaxies are sufficient to sustain the observed SFR in them for almost yet another Hubble time, $0.75 H_{0}^{-1}$. With the correction due to He and H II content taken into account this quantity increases to $1.4 H_{0}^{-1}$.

(c) The distribution of specific SFR per unit baryonic mass, $b S F R$, for late-type galaxies has rather well-defined upper limit similar to the Eddington limit for stellar luminosity. This limit is evidently determined by hard feedback: a strong burst of star formation exhausts local reserves of neutral gas thereby suppressing further process. The sample of 570 galaxies considered contains no galaxies whose $b S F R_{\mathrm{FUV}}$ would be greater than -9.5 dex. The $N\left(\log \left[b S F R_{\mathrm{H} \alpha}\right]\right)$ diagram contains two compact HII-regions: PGC 2448110 and JKB 83, and two compact Markarian galaxiesMrk 36 and Mrk 475-with $b S F R_{\mathrm{H} \alpha}$ somewhat greater than the above limit. This excess, which can be seen in the $\mathrm{H} \alpha$ line for about $10 \mathrm{Myr}$, diffuses over a time scale of about $100 \mathrm{Myr}$, which is typical for the FUV based SFR estimate.

Our $\mathrm{H} \alpha$ survey of LV galaxies and the FUV survey of these galaxies performed with GALEX telescope proved to be rather sensitive for determination of SFR for $\sim 90 \%$ late-type galaxies $\mathrm{T}=10-6$.

\section{CONCLUSIONS}

The data presented in this paper lead us to conclude that star-formation processes in irregular dwarf galaxies and disks of late-type spiral galaxies have much in common. Most of the Scd-ScSdm galaxies without apparent manifestations of a bulge must have never undergone merging acts over about the last 10 Gyr [45]. Such "virgin" galaxies are characterized by regular, sluggish SFR. Dwarf irregular galaxies have about the same average SFR, however, SFR variations among them are the higher the smaller is the baryonic mass of the dwarf. The SFR and its variations in latetype galaxies are mostly determined by individual parameters of these galaxies and depend little on external influences. In the process of the $\mathrm{H} \alpha$ survey we found a number of interesting objects where star formation appears to be caused by external factors, namely, by the onflow of intergalactic gas [46].

The distribution of specific star-formation rate per unit baryonic mass, $b S F R$, for late-type galaxies has a rather well-defined upper limit similar to the Eddington limit for stellar luminosity, which must be determined by the presence of hard feedback: a strong burst of star formation in a galaxy exhausts local reserves of neutral gas thereby suppressing further process of the birth of stars. 


\section{ACKNOWLEDGMENTS}

We are grateful to the Reviewer for careful examination of the paper and the comments that contributed to improving the paper.

This work was supported by the Russian Science Foundation (grant No. 14-02-00965).

1. I. D. Karachentsev, S. S. Kaisin, and E. I. Kaisina, Astrophysics 58, 453 (2015).

2. I. D. Karachentsev, D. I. Makarov, and E. I. Kaisina, Astron. J. 145, 101 (2013).

3. E. I. Kaisina, D. I. Makarov, I. D. Karachentsev, and S. S. Kaisin, Astrophysical Bulletin 67, 115 (2012).

4. P. A. James, N. S. Shane, J. E. Beckman, et al., Astron. and Astrophys. 414, 23 (2004).

5. D. A. Hunter and B. G. Elmegreen, Astron. J. 128, 2170 (2004).

6. R. C. Kennicutt, Jr., J. C. Lee, J. G. Funes, et al., Astrophys. J. Suppl. 178, 247 (2008).

7. A. Gil de Paz, S. Boissier, B. F. Madore, et al., Astrophys. J. Suppl. 173, 185 (2007).

8. V. L. Afanasiev, E. B. Gazhur, S. R. Zhelenkov, and A. V. Moiseev, Bull. Spec. Astrophys. Obs. 58, 90 (2005).

9. R. C. Kennicutt, Jr., Astrophys. J. 272, 54 (1983).

10. E. F. Schlafly and D. P. Finkbeiner, Astrophys. J. 737, 103 (2011).

11. R. C. Kennicutt, Jr., Annual Rev. Astron. Astrophys. 36, 189 (1998).

12. J. C. Lee, R. C. Kennicutt, Jr., S. J. J. G. Funes, et al., Astrophys. J. 692, 1305 (2009).

13. M. A. W. Verheijen, Astrophys. J. 563, 694 (2001).

14. E. J. Tollerud, M. C. Geha, J. Grcevich, et al., Astrophys.J.Lett. 798, L21 (2015).

15. E. J. Tollerud, M. C. Geha, J. Grcevich, et al., Astrophys. J. 827, 89 (2016).

16. M. P. Haynes, R. Giovanelli, A. M. Martin, et al., Astron. J. 142, 170 (2011).

17. I. D. Karachentsev, P. Riepe, T. Zilch, et al., Astrophysical Bulletin 70, 379 (2015).

18. B. P. M. Laevens, N. F. Martin, R. A. Ibata, et al., Astrophys.J.Lett. 802, L18 (2015).

19. J. T. A. de Jong, H.-W. Rix, N. F. Martin, et al., Astron. J. 135, 1361 (2008).

20. D. Kim, H. Jerjen, D. Mackey, et al., Astrophys.J.Lett. 804, L44 (2015).

21. D. R. Saul, J. E. G. Peek, J. Grcevich, et al., Astrophys. J. 758, 44 (2012).

22. J. L. Carlin, D. J. Sand, P. Price, et al., Astrophys.J.Lett. 828, L5 (2016).

23. R. B. Tully and J. R. Fisher, Astron. and Astrophys. 54, 661 (1977).

24. S. M. Faber and D. Burstein, in Large-scale motions in the universe: A Vatican study Week, Ed. by V. C. Rubin and G. V. Coyne (Princeton University, 1988), pp. 115-167.

25. B. L. James, S. E. Koposov, D. P. Stark, et al., Monthly Notices Royal Astron. Soc. 465, 3977 (2017).

26. J. N. Burchett, T. M. Tripp, J. K. Werk, et al., Astrophys.J.Lett. 779, L17 (2013).

27. B. A. Jacobs, L. Rizzi, R. B. Tully, et al., Astron. J. 138, 332 (2009).

28. A. V. Zasov, A. S. Saburova, O. V. Egorov, and R. I. Uklein, Monthly Notices Royal Astron. Soc. 469, 4370 (2017).

29. K. C. Chambers, E. A. Magnier, N. Metcalfe, et al., arXiv:1612.05560 (2016).

30. S. Danieli, P. van Dokkum, A. Merritt, et al., Astrophys. J. 837, 136 (2017).

31. I. D. Karachentsev, E. I. Kaisina, and O. G. Kashibadze Nasonova, Astron. J. 153, 6 (2017).

32. V. E. Karachentseva and I. D. Karachentsev, Astron. and Astrophys. Suppl. 127, 409 (1998). 
33. J. Koda, M. Yagi, Y. Komiyama, et al., Astrophys.J.Lett. 802, L24 (2015).

34. W. K. Huchtmeier, I. D. Karachentsev, V. E. Karachentseva, and M. Ehle, Astron. and Astrophys. Suppl. 141, 469 (2000).

35. E. Ricciardelli, A. Cava, J. Varela, and V. Quilis, Monthly Notices Royal Astron. Soc. 445, 4045 (2014).

36. I. D. Karachentsev, E. I. Kaisina, and D. I. Makarov, Monthly Notices Royal Astron. Soc. 479, 4136 (2018).

37. E. D. Skillman, New Astron. Reviews 49, 453 (2005).

38. G. S. Stinson, J. J. Dalcanton, T. Quinn, et al., Astrophys. J. 667, 170 (2007).

39. I. D. Karachentsev and S. S. Kaisin, Astron. J. 133, 1883 (2007).

40. K. B. W. McQuinn, E. D. Skillman, J. M. Cannon, et al., Astrophys. J. 695, 561 (2009).

41. I. D. Karachentsev and E. I. Kaisina, Astron. J. 146, 46 (2013).

42. G. R. Meurer, O. I. Wong, J. H. Kim, et al., Astrophys. J. 695, 765 (2009).

43. F. M. Audcent-Ross, G. R. Meurer, O. I. Wong, et al., Monthly Notices Royal Astron. Soc. 480, 119 (2018).

44. M. Fukugita and P. J. E. Peebles, Astrophys. J. 616, 643 (2004).

45. J. Kormendy, N. Drory, R. Bender, and M. E. Cornell, Astrophys. J. 723, 54 (2010).

46. A. Moiseev, I. Karachentsev, and S. Kaisin, Monthly Notices Royal Astron. Soc. 403, 1849 (2010). 